POS $\quad$ PROCEEDINGS

\title{
The X/Ka-band 2018b Celestial Reference Frame
}

\author{
Cristina García-Miró \\ Square Kilometre Array Organisation (SKAO), Jodrell Bank Observatory, \\ Lower Withington, Macclesfield, Cheshire SK11 9DL, United Kingdom \\ E-mail: c.garcia-mirolskatelescope.org
}

\section{C.S. Jacobs, J.E. Clark, L.A. White}

Jet Propulsion Laboratory, Caltech/NASA, USA

E-mail: Christopher.S.Jacobs@jpl.nasa.gov

\section{S. Horiuchi, L.G. Snedeker, I. Sotuela}

Canberra/Goldstone/Madrid DSCC/NASA, CSIRO/SAITech/ISDEFE, Australia/USA/Spain

\section{Mercolino}

ESTEC, ESA, The Netherlands

\section{B. Garcia}

CONICET, Mendoza, Argentina

\section{Colazo}

CONAE, Cordoba, Argentina

Observations at $\mathrm{X} / \mathrm{Ka}$-band are motivated by their ability to access more compact source morphology and reduced core shift relative to observations at the historically standard S/X-band. In addition, the factor of four increase in interferometer resolution at Ka-band should resolve out some extended source structure. Given these motivations, an X/Ka-band (8.4/32 GHz) celestial reference frame has been constructed using a combined NASA and ESA Deep Space Network. In 176 observing sessions we detected 678 sources covering the full 24 hours of right ascension, $\alpha$, and the full range of declinations, $\delta$. The resulting XKa median precision is now $67 \mu$ as in $\alpha \cos \delta$ and $95 \mu$ as in $\delta$.

Comparison of $529 \mathrm{X} / \mathrm{Ka}$ sources in common with the S/X-band (2.3/8.4 GHz) ICRF3 produced wRMS agreement of better than $165 \mu$ as. There is evidence for systematic errors at the $100 \mu$ as level. Known errors include limited SNR, lack of phase calibration, troposphere mismodelling, and terrestrial frame distortions. Actions are underway to reduce all of these errors. In particular, a collaboration between NASA and the ESA deep space antenna in Malargüe, Argentina is reducing weaknesses in the southern hemisphere. By comparing coordinate estimates, we probe the accuracy limits of current celestial frames in an effort to understand the advantages of each frame.

14th European VLBI Network Symposium \& Users Meeting (EVN 2018)

8-11 October 2018

Granada, Spain 


\section{The Ka-band Network}

The Ka-band network is composed NASA and ESA $\approx 35 \mathrm{~m}$ deep space telescopes (Fig. 1) including some almost orthogonal baselines and good North-South coverage. The strong geometry makes this network a powerful tool for studying systematic effects in global astrometry.

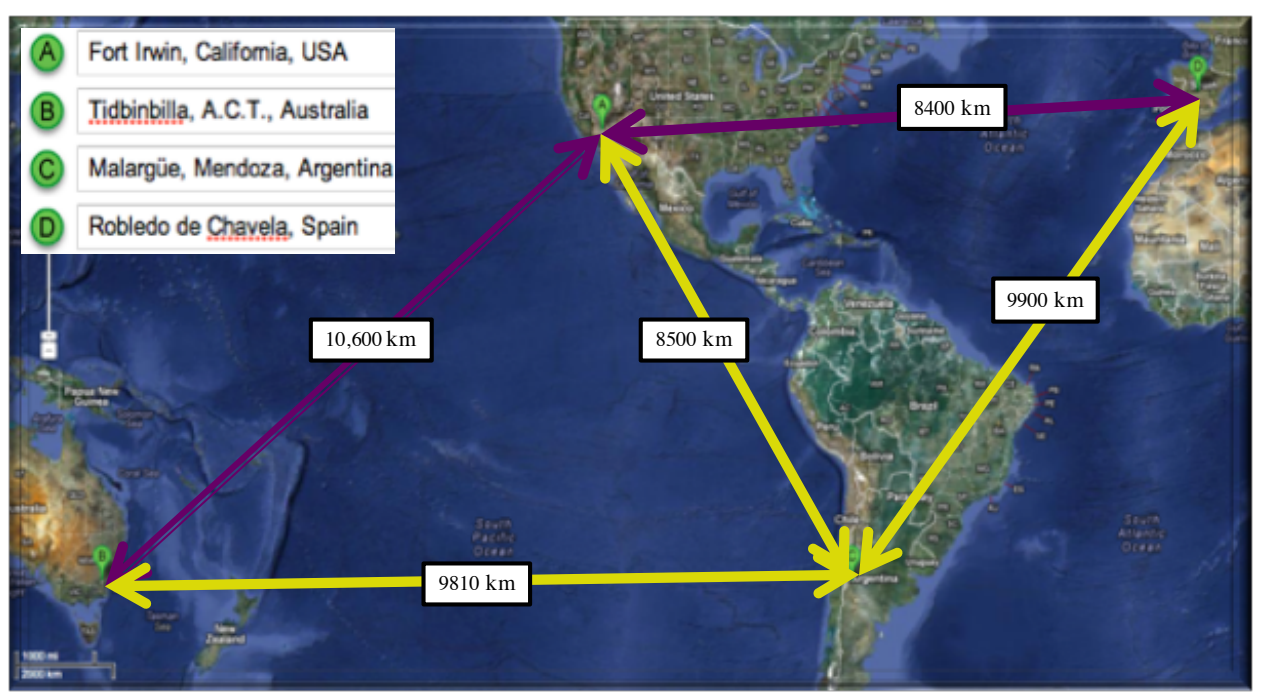

Figure 1: NASA-ESA Ka-band network. Argentina creates 3 more baselines which enable full sky coverage. Credit: Google maps.

\section{High Frequency Radio Frames}

There are several advantages of X/Ka-band compared to S/X-band for observing Active Galactic Nuclei (AGN). As the observing frequency increases, sources tend to be core dominated as the extended structure in the jets fade away (Fig. 2, left). The spatial offset of the emissions from the AGN engine due to opacity effects ("core shift") is also reduced as frequency increases (Fig. 2, right). Therefore the sources are more stable and compact. The ionosphere and solar plasma effects are also 15 times smaller. But not everything is good news. There are also disadvantages of using higher observing frequencies. The observations are more sensitive to the weather and coherence times are shorter, the sources are weaker and many are resolved, and the antenna pointing is more challenging at higher frequencies. All these effects combine to reduce the sensitivity, but one can compensate by increasing the data rates, as we have done, by increasing data rates to 2 Gbps.

\section{Accuracy: $\mathrm{X} / \mathrm{Ka}$ vs. $\mathrm{S} / \mathrm{X}$}

Comparison of the 2018b X/Ka-band solution (date 2018 Sep 28) to the current ICRF3-S/X gives 529 sources in common after removing outliers with $>5-\sigma$. The wRMS agreement is 151/ $163 \mu$ as in $\alpha \cos \delta$ and $\delta$, respectively. We tested for spatially correlated differences by estimating vector spherical harmonics (Mignard \& Klioner, 2012 [6]) to degree and order 2. The largest terms 


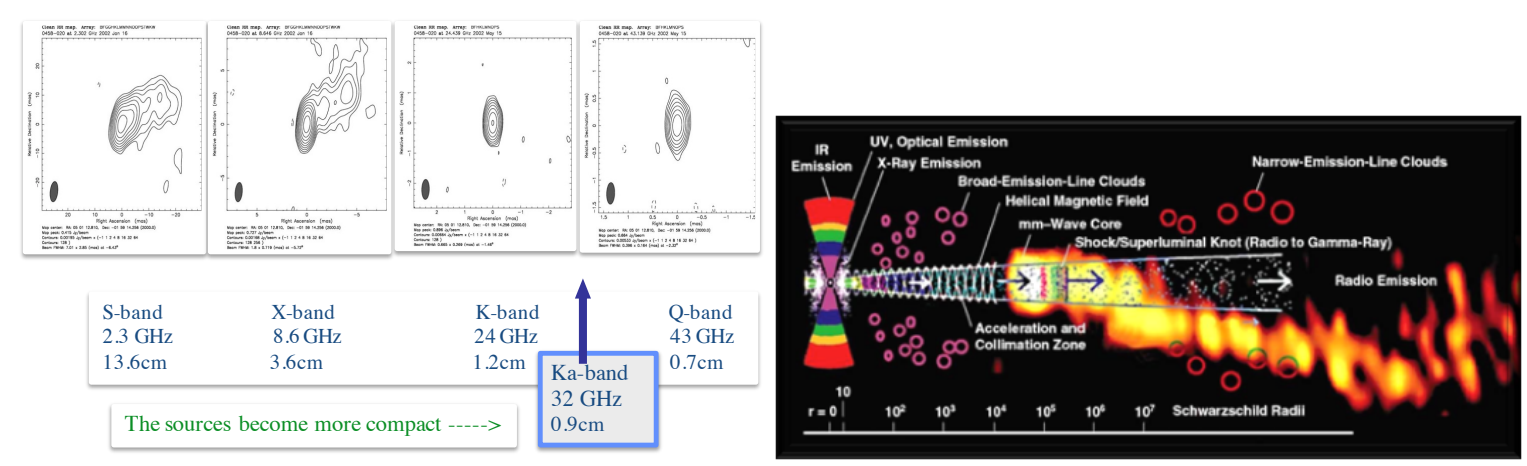

Figure 2: Left: Source structure \& compactness vs. wavelength (Charlot+, 2010 [1], Pushkarev+, 2012 [2]). Right: Schematic of Active Galactic Nuclei (Marscher, 2006 [3], Krichbaum, 1999 [4], Wehrle, 2010 [5]).

were a Z-dipole at $-183 \pm 55 \mu$ as and a quadrupole 2,0 Magnetic term at $208 \pm 20 \mu$ as. More California-Argentina baseline data will be needed to control these errors (Fig. 3, 4 and 5).
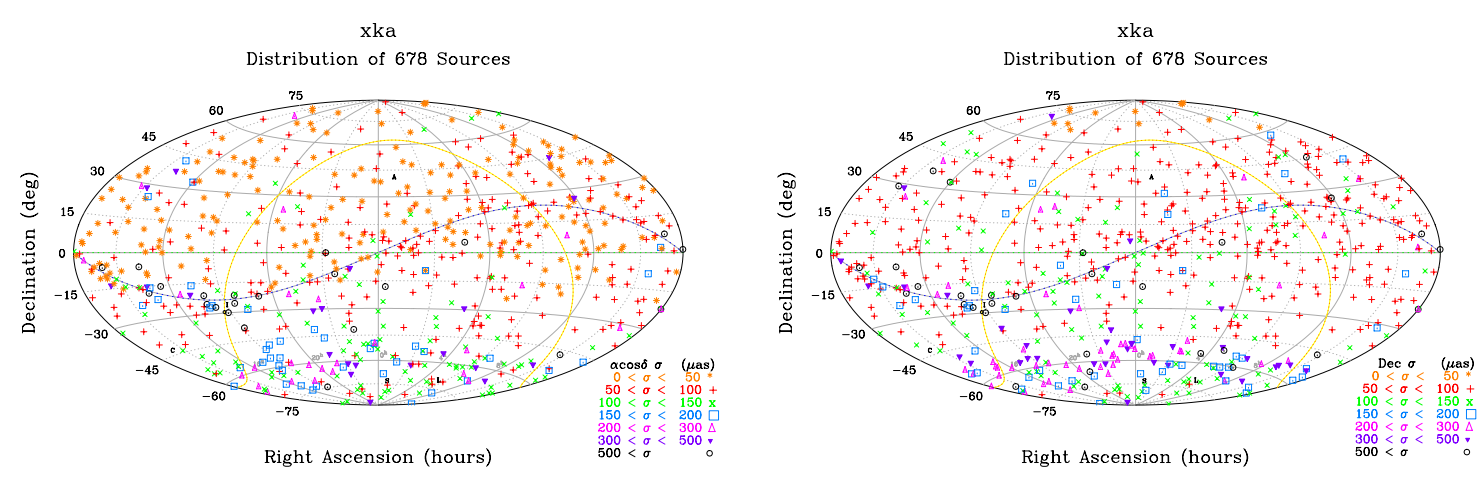

Figure 3: Left: RA* (arc) precision: Median $\sigma$ is $67 \mu$ as for 678 sources. Only 1 baseline for $\delta<-45^{\circ}$. Right: Dec precision: Median $\sigma$ is $95 \mu$ as for 678 sources. Note worse precision for $\delta<-45^{\circ}$.

\section{Gaia Optical-Radio Frame Tie and Accuracy Verification}

Launched in Dec. 2013, ESA's Gaia mission measures positions, proper motions and parallaxes of 1.7 billion objects down to magnitude 20.7, as well as photometric and radial velocity measurements. Gaia's observations will include more than 500,000 AGN of which $\sim 20,000$ will be optically bright $(V<18 \mathrm{mag})$.

The Gaia celestial frame is independent from X/Ka-band in three key respects: optical vs. radio, space vs. ground, pixel centroiding vs. interferometry. As a result Gaia provides the most independent check of accuracy available today for the radio based celestial reference frames. With Gaia Data Release-2 (Mignard+, 2018 [7]), 499 sources are detected in both the optical and X/Kaband radio, after removing 9\% (45) of the sources as outliers with $\geq 5-\sigma$ (Fig. 4, right). Rotational alignment is made with $\sim 20 \mu$ as precision (1- $\sigma$, per 3-D component). Scatter is $\sim 270 \mu$ as 

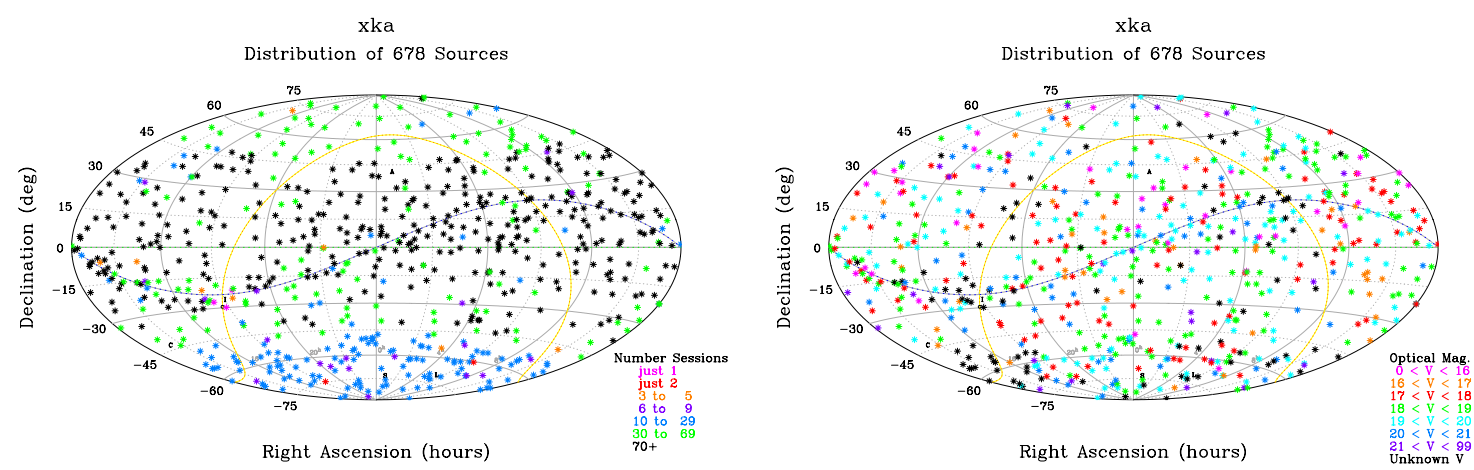

Figure 4: Left: Number of sessions: Median number of sessions is 74, but only 13 in far south. Right: Optical magnitude: Median $V=18.8$. About $30 \%$ sources bright enough for Gaia, $(V<18)$.
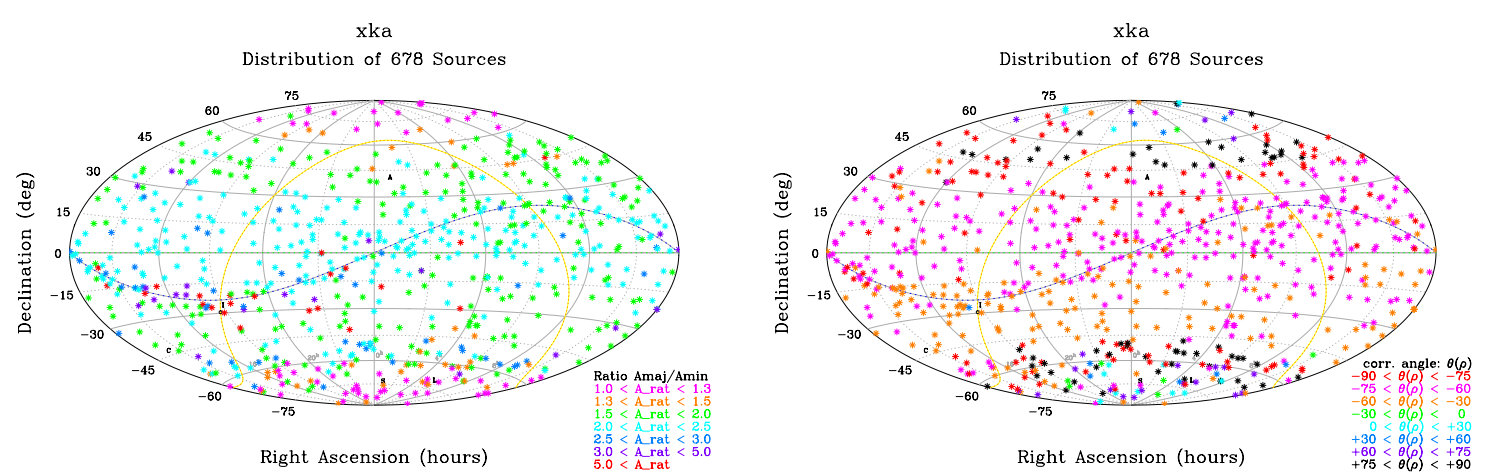

Figure 5: Left: Error Ellipse ratio $A_{m a j} / A_{\min }$ shows steady elongation from $\delta+90$ to $-45 \mathrm{deg}$. Right: Direction of Error Ellipses: semi-major axes are mostly North-South i.e. $\delta$ weaker than $\alpha$.

wRMS. Vector Spherical Harmonic difference terms out to degree and order 2 are all $210 \mu$ as or less, indicating good global agreement of the two frames.

\section{Goals for the Future}

Our first goal is to increase the number of sources, from 700 to 1000 sources, with greater density along the ecliptic plane to support navigation of interplanetary missions. As a second goal, the precision needs to be improved to $\leq 70 \mu$ as $(1-\sigma)$ to match anticipated Gaia results. In order to control the systematic errors, better uniformity is required in the observations. Thus we need to collect more data with the baselines from Malargüe, Argentina to Australia, California and Spain. Our last goal is to improve the optical-radio frame tie by adding at least 30 optically bright sources. 


\section{Conclusions}

Celestial angular coordinates $(\alpha, \delta)$ are derived from VLBI measurements at 8.4/32 GHz (36/ $9 \mathrm{~mm}$ ) of Active Galactic Nuclei. Agreement with $\mathrm{S} / \mathrm{X}$ is at the part per billion level. X/Ka has the advantage of reduced astrophysical systematics vs. S/X.

The X/Ka-band Celestial Reference Frame has 678 sources covering the full sky and is making rapid improvements in the precision. The median precision is $67 / 95 \mu$ as in $\alpha \cos \delta / \delta$. Spherical harmonic differences vs. ICRF3-S/X are $\leq 210 \mu$ as and differences vs. Gaia are $\leq 210 \mu$ as. Improving accuracy will depend on controlling systematic errors via increasing the number of observations using a North-South baseline geometry.

\section{Acknowledgments}

Thanks to all those who assisted in the data acquisition. This research was carried out in part at the Jet Propulsion Laboratory, California Institute of Technology, under a contract with the National Aeronautics and Space Administration. Support of ESA acknowledged. Argentina host country time under CONICET/CONAE sponsorship. Copyright (C)2018. All Rights Reserved.

\section{References}

[1] P. Charlot, et al., CRF at $24 \& 43$ GHz II. Imaging, AJ, 139, 2010.

[2] A.B. Pushkarev \& Y.Y. Kovalev, Single Epoch VLBI imaging of bright AGN at 2 and $8 \mathrm{GHz}$, A\&A, 544, 2012.

[3] A.P. Marscher, The Intimate Connection between High and Low Frequency Emission in Blazars, ChJAS, 6, 262, 2006.

[4] T.P. Krichbaum, A. Witzel, J.A. Zensus, 'High Angular Resolution Monitoring of Prominent AGN at 86 GHz,' eds. A. Greve \& T.P. Krichbaum, 2nd millimeter-VLBI science workshop, IRAM, Granada, Spain, 27-29 May 1999.

[5] A. Wehrle, et al, Submillimeter, Near-Infrared and Optical Light Curves of Gamma-Ray Blazars, AAS Meeting \#215, id.434.05; BAAS, 42, p.375, 2010.

[6] F. Mignard, S. Klioner, Analysis of astrometric catalogues with vector spherical harmonics, A\&A, 547, 2012.

[7] F. Mignard, S. Klioner, Gaia DRI compared to VLBI positions, Astrometry and Astrophysics in the Gaia sky, Proc. IAU Symposium 330, pp. 71-74, 2018. 